\title{
Homologies in human and Macaca fuscata chromosomes revealed by in situ suppression hybridization with human chromosome specific DNA libraries
}

\author{
J. Wienberg ${ }^{1, *}$, R. Stanyon ${ }^{2}$, A. Jauch ${ }^{1}$, and T. Cremer ${ }^{1}$ \\ ${ }^{1}$ Institut für Humangenetik und Anthropologie, Im Neuenheimer Feld 328, W-6900 Heidelberg, Federal Republic of Germany \\ ${ }^{2}$ Istituto di Antropologia, Universita Genoa, Via Balbi 4, I-Genoa, Italy \\ Received August 14, 1991 \\ Accepted September 12, 1991 by E.R. Schmidt
}

\begin{abstract}
We established chromosomal homologies between all chromosomes of the human karyotype and that of an old world monkey (Macaca fuscata) by chromosomal in situ suppression (CISS) hybridization with human chromosome specific DNA libraries. Except for the human chromosome 2 library and limited cross-hybridization of $\mathrm{X}$ and $\mathrm{Y}$ chromosome libraries all human DNA libraries hybridized to single GTG-banded macaque chromosomes. Only three macaque chromosomes $(2,7,13)$ were each hybridized by two separate human libraries ( 7 and 21,14 and 15,20 and 22 respectively). Thus, an unequivocally high degree of synteny between human and macaque chromosomes has been maintained for more than 20 million years. As previously suggested, both Papionini (macaques, baboons, mandrills and cercocebus monkeys, all of which have nearly identical karyotypes) and humans are chromosomally conservative. The results suggest, that CISS hybridization can be expected to become an indispensable tool in comparative chromosome and gene mapping and will help clarify chromosomal phylogenies with speed and accuracy.
\end{abstract}

\section{Introduction}

Knowledge of chromosomal homology with other primates is essential to determine the origin of human chromosomes, to understand the mode of chromosomal evolution, to reconstruct phylogenies and finally to clarify the role of chromosomal rearrangements in speciation. Comparative cytogenetics has always been limited by difficulties in establishing between-species chromosomal homology. Chromosomal homology has usually been based on comparative banding and gene mapping or a combination of these two methods. Between humans

\footnotetext{
* Present address: Institut für Anthropologie und Humangenetik, Richard-Wagner-Strasse 10, W-8000 München, Federal Republic of Germany

Offprint requests to: J. Wienberg
}

and great apes there is a well known correspondence between chromosomal banding and gene mapping with just a few exceptions (O'Brien et al. 1988; Seuànez 1987). For many other species gene mapping data are absent or contradictory and homology has been suggested so far only by subjectively matching banding patterns. Alternatively, gene mapping data have suggested the synteny of genes, while chromosomal banding was not informative (cf. O'Brien and Graves 1990). Because gene mapping has a higher resolution and can establish homologies directly at the level of DNA sequences it is usually given priority in comparative karyotype analysis (Ruddle 1981). However, the comparative localization of individual genes provides only patchy information on chromosome homology.

Gene mapping and chromosome banding studies have provided conflicting data for the chromosomes of Papionini (Créau-Goldberg et al. 1983; Seuànez 1987). Unequivocal data for macaque/human chromosome homologies would be particularly desirable, because Papionini have a conservative karyotype which appears to be essentially the same in all these species (Stanyon et al. 1983, 1990). Accordingly, these species can be used as an 'outgroup' for determining the direction of chromosome rearrangements in great ape and human evolution.

Recently, we have applied chromosomal in situ suppression (CISS) hybridization of DNA libraries established from flow sorted human chromosomes (Cremer et al. 1988; Lichter et al. 1988; Pinkel et al. 1988) as a new approach to establish extended chromosomal homologies on the basis of DNA sequence homologies (Wienberg et al. 1990). In this study we used this approach for comparative chromosome mapping of the entire human and Macaca fuscata karyotypes and present the first complete analysis of interchromosomal rearrangements that have occurred since the divergence of the human and a monkey karyotype. 


\section{Materials and methods}

Chromosome preparations. Chromosome preparations from $M . f u s-$ cata were prepared and stored as previously reported (Jauch et al. 1990; Stanyon et al. 1990). Routine trypsin G-banding was performed prior to CISS hybridization. Banded metaphases were photographed (Agfa-Ortho 25), destained with fixative (3:1 methano-1: acetic acid) and postfixed with $4 \%$ formaldehyde (Merck) in PBS for $15 \mathrm{~min}$ as described by Klever et al. (1991). Postfixation was essential to preserve chromosome morphology in the subsequent CISS hybridization experiments. All hybridized macaque chromosomes were identified on the basis of their trypsin G-banding pattern (see Fig. 1d, f, i).

CISS hybridization. CISS hybridization to primate chromosomes was performed as previously described (Wienberg et al. 1990). Human chromosome specific DNA libraries established from flow sorted human chromosomes and cloned into a bacteriophage vector (American Type Culture Collection no. LA01 NS01, LL02NS01, LA03NS02, LA04NS02, LA05NS01, LL06NS01, LA07NS01, LL08NS02, LL09NS01, LL10NS01, LL11NS01, LA12NS01, LL13NS02, LL14NS01, LL15NS01, LL16NS03, LL17NS02, LL18NS01, LL19NS01, LL20NS01, LA21NS01, LL22NS01, LA0XNS01, LLOYNS01) or subcloned into a plasmid vector (pBS1-pBS4, pBS6-pBS22, pBSX, pBSY) were used as probes. Plasmid libraries are described in detail by Collins et al. (1991) and were kindly provided by J. Gray, Livermore, USA. $10 \mu$ standard hybridization mixture containing $2 \mu \mathrm{g}$ labelled phage library DNA or $500 \mathrm{ng}$ labelled plasmid library DNA was placed on slides and mounted with $18 \mathrm{~mm} \times 18 \mathrm{~mm}$ coverslips.

Detection of hybridization signals. Biotinylated DNA probes were detected with fluorescein isothiocyanate (FITC) coupled with avidin (Vector Laboratories). Double hybridization experiments were performed with digoxigenin labelled probes, which were detected with FITC labelled mouse anti-digoxigenin antibodies (Boehringer Mannheim), together with biotin labelled probes, which were detected with avidin coupled to AMCA (7-amino-4-methylcoumarin3-acetic acid, Vector Laboratories). Signals were amplified once as described by Pinkel et al. (1986). Chromosome preparations were counterstained with propidium iodide and embedded with an antifade as described (Jauch et al. 1990). Slides were analysed with a Zeiss photomicroscope III equipped for FITC and AMCA fluorescence. Photographs were taken on Agfachrome $1000 \mathrm{RS}$ (1000 ASA) or Kodak T-MAX black and white (400 ASA) films. For chromosome breakpoint analysis the photographic slides from CISS hybridization and GTG-banding of the same metaphase plate were superimposed on a screen.

\section{Results}

Typical hybridization patterns obtained with human chromosome specific DNA libraries on GTG-banded macaque chromosomes are shown in Fig. 1. The macaque idiogram presented in Fig. 2 summarizes the homologies of human and macaque chromosomes based on CISS hybridization patterns of all 24 human chromosome DNA libraries. DNA libraries from 15 human autosomes each hybridized to one macaque homolog (e.g. Fig. $1 \mathrm{a}, \mathrm{c})$. The human chromosome 2 DNA library was the only that showed labelling (with the exception of limited cross-hybridization of $\mathrm{X}$ and $\mathrm{Y}$ chromosome libraries) on two macaque chromosomes, 9 and 15 (Fig. 1 b). Three macaque autosomes showed extended signals with two human autosome libraries indicating that these macaque chromosomes were derived either by fusion events or that the two human homologous had evolved by fission of the single ancestral chromosome. Macaque chromosome 2 hybridized with both human chromosome 7 and 21 libraries (not shown), while macaque chromosome 7 (Fig. $1 \mathrm{f}$, arrow) hybridized with libraries of human chromosomes 14 (not shown) and 15 (Fig. 1 e). Macaque chromosome 13 (Fig. 1i, arrowheads), the marker chromosome bearing the nucleolar organizer region (NOR), was "painted" by probes from human chromosomes 20 (Fig. $1 \mathrm{~h}$, arrow) and 22 (Fig. $1 \mathrm{~g}$, arrow). G-banding combined with subsequent double hybridization and two colour detection of differentially labelled DNA probes (biotin or digoxigenin) allowed a more precise mapping of the hybridization signal on these macaque chromosomes (Fig. $1 \mathrm{~g}-\mathrm{i}$ ).

The human $Y$ chromosome library labelled the entire macaque $Y$ chromosome, but also showed cross-hybridization to the pseudoautosomal region of the $\mathrm{X}$ chromosome, to a band presumably homologous to human Xq1.3, and - unexpectedly - to two bands located on macaque chromosomes 6 and 15 (Fig. 2). The human $\mathrm{X}$ chromosome library in addition to the painting of the entire macaque $X$ chromosome showed cross-hybridization to a macaque Yq band close to the centromere (not shown). The libraries for chromosomes 13, 14, 15, 21 and 22 , which cross-hybridize on human chromosomes near the centromeres of all members of this group (Collins et al. 1991), only hybridize to a single macaque chromosome each.

Fig. 1a-i. Macaque metaphase spreads demonstrate chromosomes "painted" by chromosomal in situ suppression (CISS) hybridization with biotin or digoxigenin labelled bacteriophage or plasmid DNA libraries from sorted human chromosomes $(\mathbf{a}, \mathbf{b}, \mathbf{c}, \mathbf{e ,} \mathbf{g}$, h). All spreads were G-banded prior to CISS hybridization to allow the identification of the painted macaque chromosomes or chromosome segments (e.g. d, f, i). Biotinylated probes were detected with avidin conjugated to fluorescein isothiocyanate (FITC; green) (a, b, c, e) or 7-amino-4-methylcoumarin-3-acetic acid (AMCA; blue) (h), or with FITC coupled antibodies against digoxigenin (g). Chromosomes were counterstained with propidium iodide (red). a The human chromosome 1 library paints the homologous macaque chromosome 1 . Note the reduced labelling over the terminal part of macaque 1q (arrowhead) (also seen in human chromosome 1p, not shown) and the lack of label on the centromeric heterochromatin (arrow). b Human chromosome 2 library delineates the two submetacentric macaque chromosomes 9 and 15 . Note the reduced labelling in the short arms of the two homologous pairs. $c$ The human chromosome 9 DNA library paints the entire long arm of macaque chromosome 11, while most of the heterochromatic short arm remains unlabelled (arrow). In contrast the human chromosome 9 is entirely labelled by this library (insert). d G-banding of the metaphase shown in $\mathbf{c}$. $\mathbf{e}$ The human chromosome 15 DNA library delineates the entire short arm and a small part of the long arm of macaque chromosome 7 (arrow). f G-banding of the metaphase shown in $\mathbf{d}$. $\mathbf{g}, \mathbf{h}$ Two colour CISS hybridization with a digoxigenin labelled human chromosome 22 library (arrow) detected with FITC (g) and a biotin labelled human chromosome 20 library (arrow) detected with AMCA (h) shows that the short arm of macaque chromosome 13 is homologous to human chromosome 22 , while most of its long arm is homologous to human chromosome 20. The nuclear organizer region (NOR) is not labelled with either library. $\mathbf{i}$ G-banding of the metaphase shown in $\mathbf{g}$ and $\mathbf{h}$; arrowheads point to the NOR 

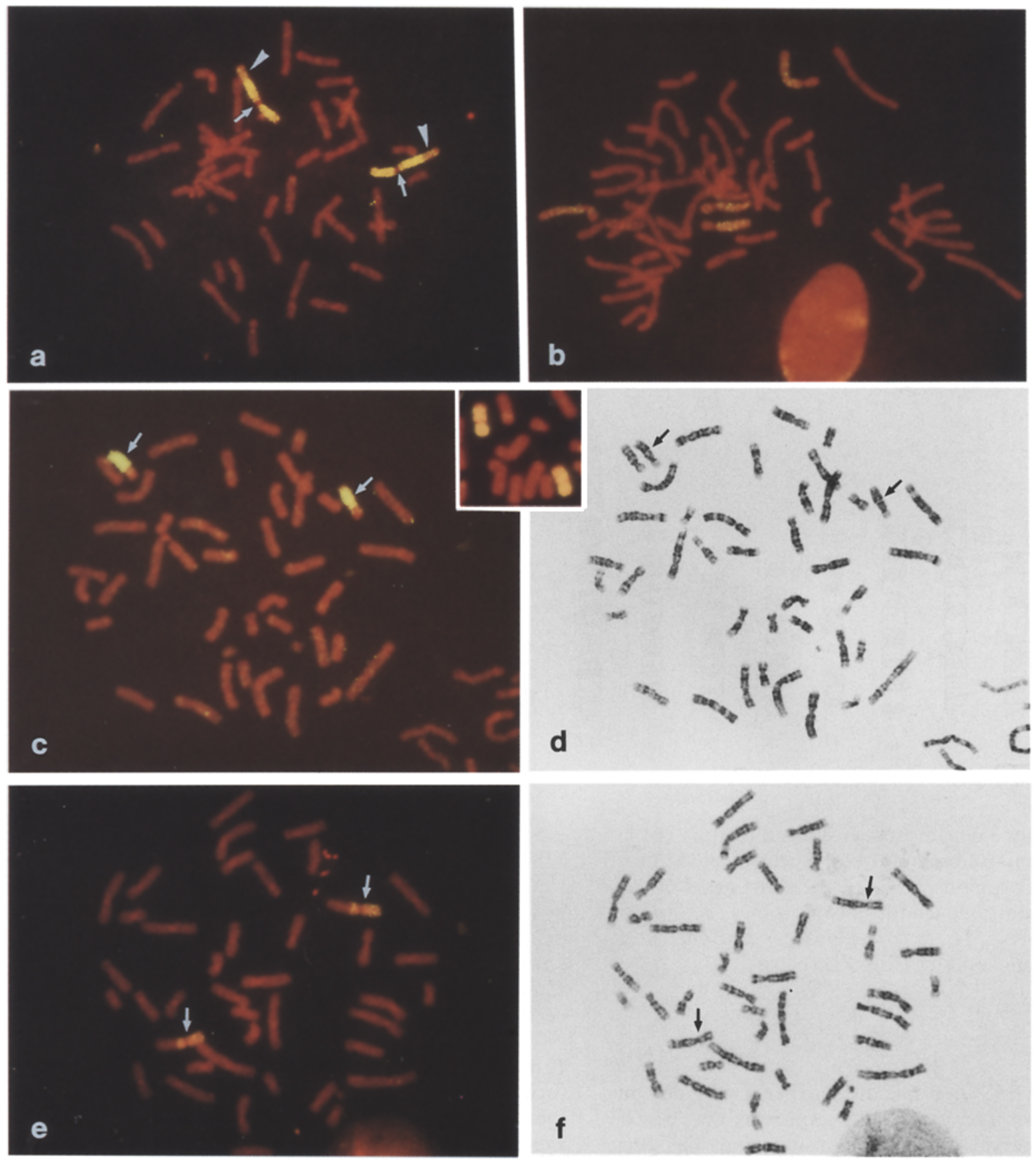

d
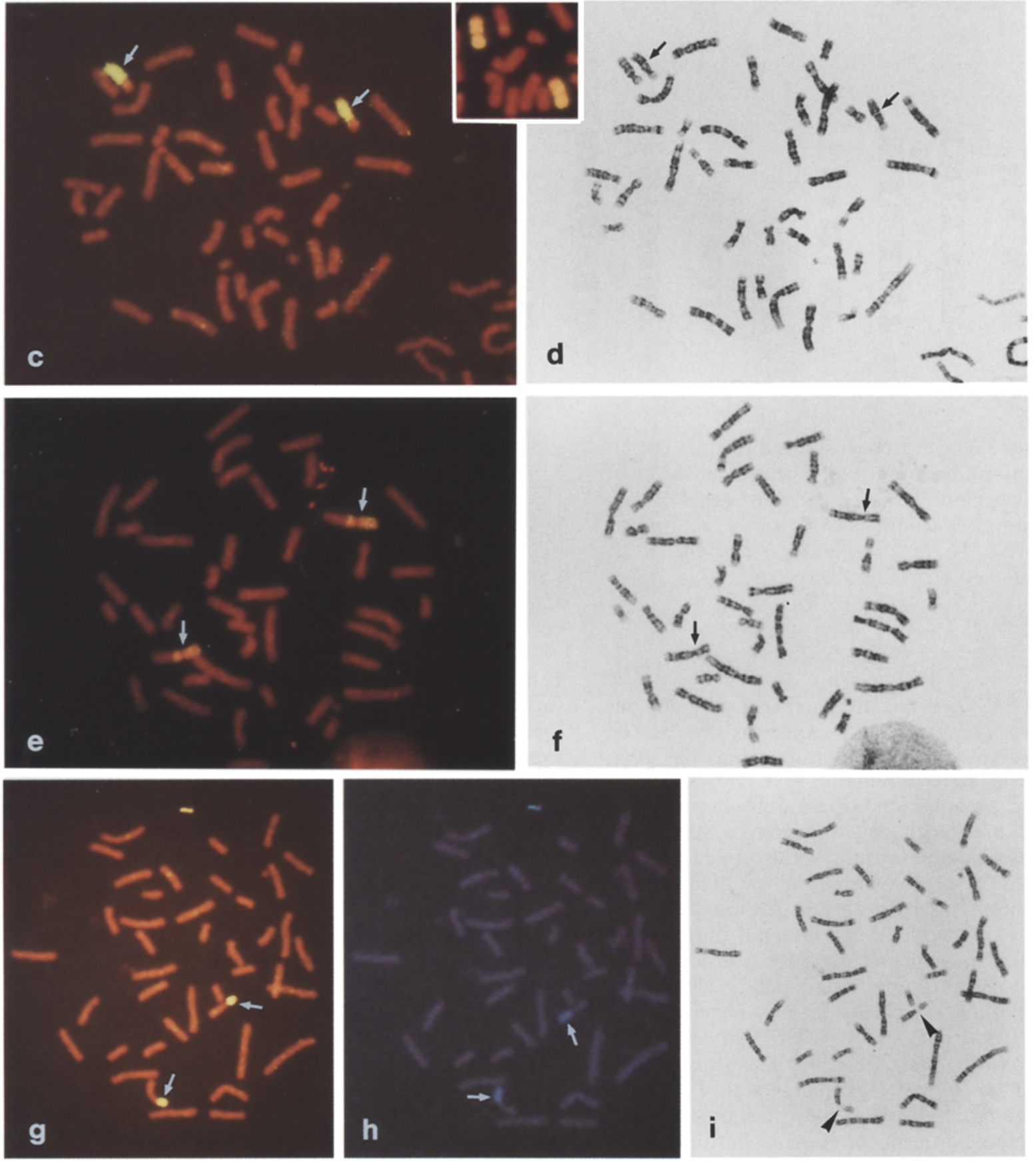

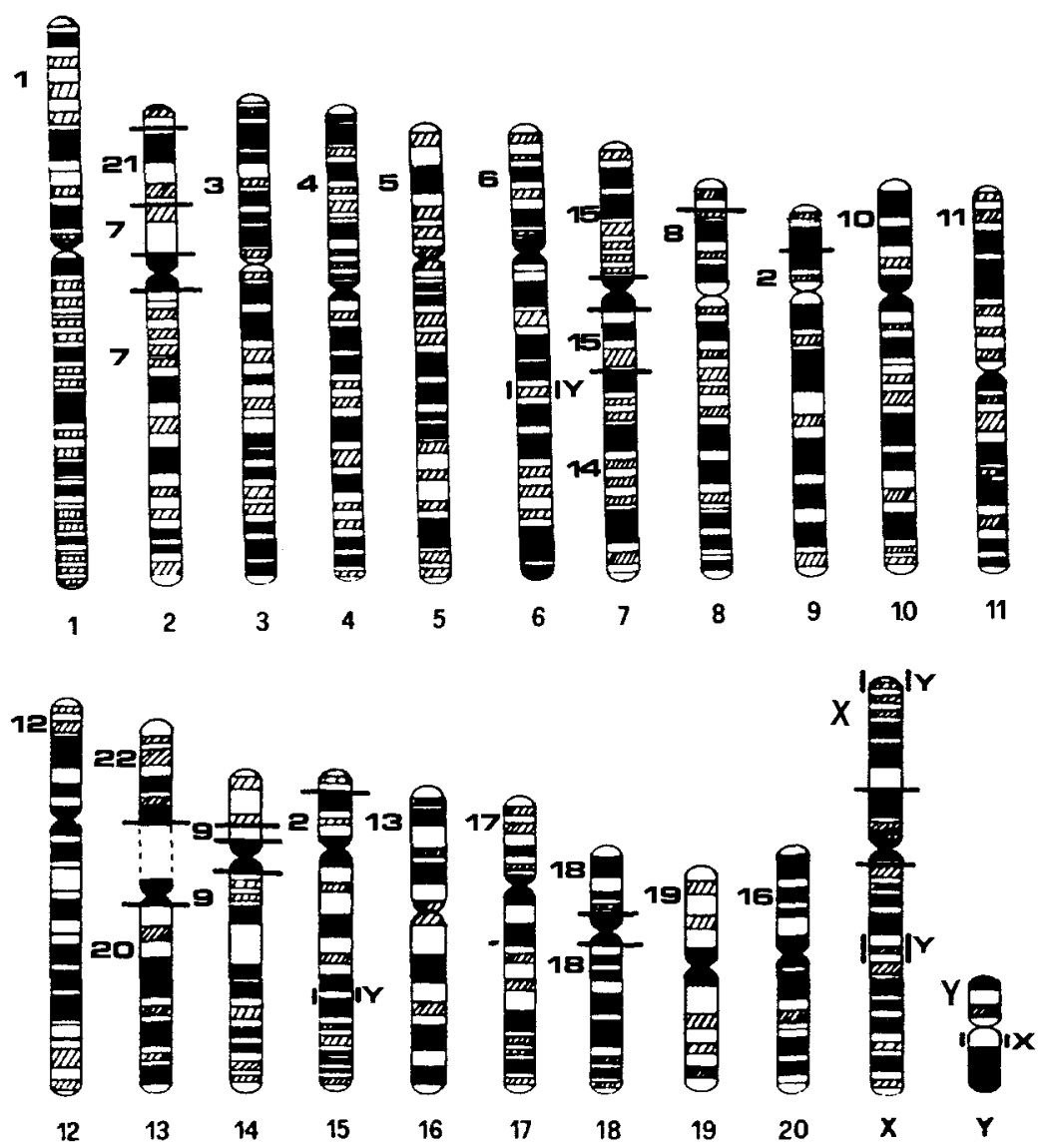

Fig. 2. Idiogramatic representation of CISS hybridization patterns from plasmid and bacteriophage DNA libraries derived from flow sorted human chromosomes hybridized to G-banded chromosomes of Macaca fuscata (idiogram after Small et al. 1985). Macaque chromosome numbers given below each chromosome; numbers on the left between bars indicate subregions painted with the respective human chromosome specific library. Regions of cross-hybridization of human chromosome $X$ and $Y$ libraries are indicated on the right

Some macaque chromosomes showed regions not labelled with human library probes. These included most pericentromeric regions, parts of the short arms of various submetacentric macaque chromosomes $(2 \mathrm{p}, 8 \mathrm{p}, 9 \mathrm{p}$, 14p, 15p; see Fig. 1b, c for examples) and the NOR region of chromosome 13 (Fig. $1 \mathrm{~g}-\mathrm{i}$ ).

\section{Discussion}

The present CISS hybridization experiments demonstrate that the synteny of DNA sequences that can be observed in individual human chromosomes has been maintained for at least 20 million years. Most macaque chromosomes showed homology to a single human chromosome. Only three macaque chromosomes showed homology with two human chromosomes. Even in these cases synteny of the genes observed in the human chromosomes has been largely preserved. Although we cannot exclude loss of some genetic material during these chromosome rearrangements, material translocated to other macaque chromosomes could not be detected in our present experiments (see also below). The only obvious exception from the conservation of synteny is provided by human chromosome 2 . As has already been shown for the great apes (Wienberg et al. 1990), the DNA library from this chromosome also shows homology with two submetacentric $M$. fuscata chromosomes. Some heterochromatic macaque chromosomal subre- gions were not labelled by human chromosome specific DNA libraries. This lack of labelling may be due to rapid divergence in repetitive sequences or to sequences not present in these DNA libraries (Collins et al. 1991).

\section{Comparative chromosome banding analysis versus CISS hybridization}

It has been asserted previously that chromosome banding studies alone provide reliable information for tracing the phylogeny of chromosomes from prosimians (or even from outside the primate order) to human (Dutrillaux 1979, 1986; Clemente et al. 1990). Our present data indicate the possible limitations of an approach based exclusively on the matching of band sequence similarities. For more than 20 years many authors have proposed homologies based on comparisons of banding patterns between the Papionini and human karyotypes. When comparing these results with those obtained in the present CISS hybridization experiments, the accuracy of the proposed autosomal homologies ranged from 1 in 4 to 16 in 22 (Stock and Hsu 1973; Dutrillaux et al. 1979; Bernstein et al. 1980; Soares et al. 1982; Stanyon and Chiarelli 1983). Comparisons between phylogenetically more distant species, which most often differ for many more rearrangements, may be even less reliable, while establishing homology by chromosome banding in species characterized by pronounced chromo- 
some shuffles (i.e. hylobatids, rodents) has been virtually impossible (O'Brien et al. 1988; Seuànez 1987).

The combination of chromosome banding procedures with CISS hybridization has now made it possible to map chromosomes comparatively at the DNA level and provides a more secure information basis for proposing chromosome homologies. In our experiments the information derived from chromosome banding patterns was used solely for the identification of the macaque chromosomes, while the decision on homologies between the human and macaque chromosomes was based solely on the CISS hybridization patterns. Superimposition of the two patterns then allowed a precise comparison.

\section{Comparative gene mapping versus CISS hybridization}

Generalization from a few patchy gene mapping data on the homology of extended regions or even complete chromosomes may be misleading because homologies between single mapped genes are extrapolations. In contrast, extended homologous regions comprising at least a few megabases can be unequivocally detected in apes and monkey chromosomes by CISS hybridization with human chromosome specific DNA libraries. For example gene mapping data for macaque chromosome 2 has so far only provided evidence for one human homolog (Estop et al. 1979) while the present CISS hybridization experiments have demonstrated homology for two human chromosomes. Problems of interpretation may occasionally occur in CISS hybridization experiments where suppression of interspersed repetitive sequences is insufficient or when cross-hybridization is present due to some tandem-repetitive sequences in common between chromosomes.

The majority of gene mapping data from macaque and baboon chromosomes are consistent with the results of the present CISS hybridization experiments. An association of human 6 and 7 syntenic groups in the macaque, however, as proposed by Estop et al. (1979) could not be supported. There are several possibilities to explain this inconsistency. Small rearranged parts of homologous chromosome material may have escaped detection by the CISS hybridization technique. On the other hand, in hybrid cell lines erroneous gene assignment may be due to chromosomal rearrangements in vitro or to difficulties in the complete assessment of chromosomes.

The GTG-banding patterns of some macaque chromosomes painted by a single human DNA library are apparently identical to those of their human homolog. In other macaque and human homologous further intrachromosomal rearrangements must be hypothesized to match banding patterns. Whole chromosome libraries identify homologous chromosome segments without giving information about additional structural changes, e.g. inversions, which may have occurred within such segments. To overcome this limitation CISS hybridization of libraries established from microdissected chromosome bands and individual DNA clones such as yeast artificial chromosomes or cosmid clones (Lengauer et al. 1991;
Lichter et al. 1990 and our unpublished data) can be applied. We expect that this approach will provide an indispensable tool for future studies of comparative chromosome and gene mapping and will help to clarify chromosomal phylogenies with speed and accuracy.

Acknowledgements. We would like to thank A. Wiegenstein and J. Hollatz for photographic work and B. Schoell, F. Bigoni and S. Tofanelli for technical help. T.C. was the recipient of a Heisenberg-Stipendium from the Deutsche Forschungsgemeinschaft. This work was supported in part by the Deutsche Forschungsgemeinschaft and the Italian CNR bilateral program 90.02476.CT04.

\section{References}

Bernstein R, Pinto M, Morcom G, Bielert C (1980) A reassessment of the karyotype of Papio ursinus: homoeology between human chromosome 15 and 22 and a characteristic submetacentric baboon chromosome. Cytogenet Cell Genet 28:55-63

Clemente IC, Ponsa M, Garcia M, Egozcue J (1990) Evolution of the Simiiformes and the phylogeny of human chromosomes. Hum Genet 84:493-506

Collins C, Kuo WL, Segraves R, Pinkel D, Fuscoe J, Gray JW (1991) Construction and characterization of plasmid libraries enriched in sequences from single human chromosomes. Genomics, in press

Créau-Goldberg N, Turleau C, Cochet C, de Grouchy J (1983) New gene assignments in the baboon and new chromosome homoeologies with man. Ann Genet 26:75-78

Cremer T, Lichter P, Borden J, Ward DC, Manuelidis L (1988) Detection of chromosome aberrations in metaphase and interphase tumor cells by in situ hybridization using chromosomespecific library probes. Hum Genet 80:235-246

Dutrillaux B (1979) Chromosomal evolution in primates: tentative phylogeny from Microcebus murinus (prosimian) to man. Hum Genet 48:251-314

Dutrillaux B (1986) Evolution chromosomique chez les primates, les carnivore et les rongeurs. Mammalia, vol 50, numero special

Dutrillaux B, Viegas-Paequignot, Dubos C, Masse R (1978) Complete or almost complete analogy of chromosome banding between the baboon (Papio papio) and man. Hum Genet 43:37-46

Estop A, Garver JJ, Meera-Khan P, Pearson PL (1979) Rhesushuman chromosome homologies via cytogenetic and gene mapping studies, Human Gene Mapping 5. Cytogenet Cell Genet 25:150-151

Jauch A, Daumer C, Lichter P, Murken J, Schroeder-Kurth T, Cremer T (1990) Chromosomal in situ suppression hybridization of human gonosomes and autosomes and its use in clinical cytogenetics. Hum Genet 85:145-150

Klever M, Grond-Ginsbach C, Scherthan H, Schroeder-Kurth T (1991) Chromosomal in situ suppression hybridization after Giemsa banding. Hum Genet 86:484-486

Lengauer C, Luedecke H-J, Wienberg J, Cremer T, Horsthemke B (1991) Comparative chromosome band mapping in primates by in situ suppression hybridization of band specific DNA microlibraries. Hum Evol 6:67-71

Lichter P, Cremer T, Borden J, Manuelidis L, Ward DC (1988) Delineation of individual human chromosomes in metaphase and interphase cells by in situ suppression hybridization using recombinant DNA libraries. Hum Genet 80:224-234

Lichter P, Chang Tang CJ, Call K, Hermanson G, Evans GA Housman D, Ward DC (1990) High resolution mapping of human chromosome 11 by in situ hybridization with cosmid clones. Science 247:64 69

O'Brien SJ, Graves JAM (1990) Human gene mapping 5; report of the committee on comparative gene mapping. Cytogenet Cell Genet 55:406-433 
O'Brien SJ, Seuànez HN, Womack JE (1988) Mammalian genome organization: an evolutionary view. Annu Rev Genet 22:323351

Pinkel D, Gray JW, Trask B, van den Engh G, Fuscoe J, van Dekken H (1986) Cytogenetic analysis by in situ hybridization with fluorescently labeled nucleic acid probes. Cold Spring Harbor Symp Quant Biol 51:151-157

Pinkel D, Landegent J, Collins C, Fuscoe J, Segraves R, Lucas J, Gray JW (1988) Fluorescence in situ hybridization with human chromosome specific libraries: Detection of trisomy 21 and translocations of chromosome 4. Proc Natl Acad Sci USA 85:9138-9142

Ruddle FH (1981) A new era in mammalian gene mapping: somatic cell genetics and recombinant DNA methodologies. Nature 294:115-119

Seuànez HN (1987) The chromosomes of man: Evolutionary considerations. In: Obe G, Basler A (eds) Cytogenetics. Springer, Berlin Heidelberg New York

Small M, Stanyon R, Smith DG, Sineo L (1985) High-resolution chromosomes of rhesus macaques (Macaca mulatta). Am J Primatol 9:63-67
Soares MBM, Armada JL, Armada A, da Silva VF, Seuànez HN (1982) Standardization of the karyotype of the rhesus monkey, Macaca mulatta, and interspecific homologies with human chromosomes. J Hum Evol 11:291-296

Stanyon R, Chiarelli B (1983) Phylogeny of the hominoidea: the chromosome evidence. J Hum Evol 11:493-504

Stanyon R, Ardito G, Lamberti L, Bigatti P (1983) The banded karyotypes of Macaca fuscata compared with Cerocebus aterrimus. Folia Primatol 41:137-146

Stanyon R, Romagno D, Wienberg J, Maurer U (1990) Sequence of DNA replication in Macaca fuscata chromosomes: An outgroup for phylogenetic comparison between man and apes. Genetica $80: 45-52$

Stock AD, Hsu TC (1973) Evolutionary conservatism in arrangement of genetic material: a comparative analysis of chromosome banding between the rhesus macaque $(2 n=42,84$ arms) and the African green monkey $(2 \mathrm{n}=60,120 \mathrm{arms})$. Chromosoma 43:211-224

Wienberg J, Jauch A, Stanyon R, Cremer T (1990) Molecular cytotaxonomy of primates by chromosomal in situ suppression hybridization. Genomics 8:347-350 